\title{
Testicular Orphan Receptor 4 (TR4) Promotes Papillary Thyroid Cancer Invasion via Activating Circ-FNLA/miR-149-5p/MMP9 Signaling
}

\section{Xiwu Ouyang}

Xiangya Hospital Central South University

Lemeng Feng

Xiangya Hospital Central South University

Lei Yao

Xiangya Hospital Central South University

Yao Xiao

Xiangya Hospital Central South University

Gewen Zhang

Xiangya Hospital Central South University

Guodong Liu

Xiangya Hospital Central South University

Zhiming Wang ( $\nabla$ wzm13808462382@126.com )

Xiangya Hospital Central South University

\section{Research}

Keywords: Thyroid cancer, TR4, Circ-FLNA, Cell invasion

Posted Date: July 17th, 2020

DOI: https://doi.org/10.21203/rs.3.rs-43637/v1

License: (c) (1) This work is licensed under a Creative Commons Attribution 4.0 International License.

Read Full License

Version of Record: A version of this preprint was published at Molecular Therapy - Nucleic Acids on June 1st, 2021. See the published version at https://doi.org/10.1016/j.omtn.2021.03.021. 


\section{Abstract}

Background: The incidence and mortality of papillary thyroid cancer (PTC) have steadily increased. Although conventional therapies are very effective towards differentiated PTC patients, very limited therapeutic options are applicable to those patients with distant metastases. Therefore, better understanding of the molecular biology of metastatic PTC helps identify novel targets and facilitates the development of new therapies.

Methods: Western blot assay was used to check relative gene protein expression. Transwell invasion assay was used to check the invasion capacity of the PTC cells. Wound healing assay was conducted to check the migration capacity of the PTC cells. qRT-PCR assay was used to check relative RNA expression of the cells. IHC assay was used to check TR4 and MMP9 expression in clinical samples or mouse tumor samples.

Results: In this study, we first found that testicular orphan receptor 4 (TR4) was significantly increased in PTC tumors spreading to lymph node compared to the paired primary tumors. Experimental evidence suggested that TR4 drove PTC progression via promoting its cell invasion and cell migration. Mechanistically, TR4 transcriptionally regulated the expression level of circ-FLNA, which competed with MMP9 for miR-149-5p binding and led to increased protein level of MMP9. Interruption assays with various gene manipulations verified that TR4/circ-FLNA/miR-149-5p/MMP9 signaling axis played central role in cell invasion and cell migration of PTC cells. Moreover, xenografted mouse model also confirmed that TR4/circ-FLNA signal promoted PTC tumor growth.

Conclusions: Overall, our study pinpoints the oncogenic role of TR4 in PTC development and targeting TR4/circ-FLNA/miR-149-5p/MMP9 signaling may be an alternative option for metastatic PTC patients.

\section{Background}

Thyroid cancer (TC) is a common endocrine malignancy worldwide [1-2]. Its incidence rate was steadily increased in the past decades. According to the global survey in 2012, approximate 300,000 new cases of thyroid cancer were diagnosed and 40,000 deaths were reported [3]. TC is usually divided into differentiated thyroid cancer (DTC), medullary thyroid cancer (MTC) and anaplastic thyroid cancer (ATC). And papillary thyroid cancer (PTC) accounts for the majority of DTC. Current treatments such as surgery, radioactive iodide therapy, and hormone-suppressive therapy show promising therapeutic efficacy in patients with PTC. However, about 30\% PTC will develop distant metastases [4-5]: lymph node, bone, lung and liver are desirable organs PTC prefers to invade. For such patients with metastasis, the mortality is rather high due to the worse 5-year survival rate (about 50\%) and limited treatment options. Therefore, better understanding of the molecular biology of metastatic PTC will accelerate the development of novel targeted therapies.

Testicular orphan receptor 4, also called TR4 or NR2C2, was first cloned from testis and was a nuclear receptor without identified ligand [6]. Early studies with TR4 knockout mice have demonstrated that TR4 
is a key factor to maintain fertility, bone development and metabolic homeostasis [7-8]. As a transcription factor, activated TR4 is translocated to the nucleus where it binds its recognized DNA element and regulates a bundle of genes. Now increasing evidence suggest that TR4 may also contribute to cancer development [9-13]. For instance, TR4 played a tumor promoting role in RCC by promoting vasculogenic mimicry formation and tumor metastasis [9]. Mechanistically, TR4 could increase the expression level of vimentin via downregulating its associated miRNA, miR490-3p. In PCa, evidence suggested that TR4 could suppress PCa initiation via regulating ATM to reduce DNA damages [12]. However, TR4 was overexpressed in PCa patients and its expression level was tightly associated with Gleason score, implying it may promote PCa progression. Indeed, in vitro and in vivo studies showed that TR4 could increase PCa invasion/metastasis via its regulation on CCL2, TGFbetaR2/Smad3 and stem cells population [11]. However, little is known about the role of TR4 in PTC carcinogenesis and progression.

Circular RNAs (circRNAs) are pre-mRNA back splicing products of exons and increasing receive interest in the field of cancer research [14-15]. The function of one certain circRNA relies on its regulation on gene expression [16]: circRNA can act as a sponge of miRNA and lead to the upregulation of its targeted genes; nuclear circRNA regulates gene expression via unknown mechanisms; finally, the processing of circRNAs will impair the alternative splicing of such pre-mRNAs and causes altered gene expression. The contributions of circRNAs to PTC development have been documented recently. For instance, circ-FOXM1 was upregulated in PTC patients and promoted PTC growth by acting as a sponge of miR-1179 to increase HMGB1 expression [17]. Evidence also demonstrated that circ-BACH2 increased PTC cell proliferation, invasion and migration by preventing miR-13p-5p from binding to the 3'UTR of LMO4 [18]. These findings imply that circRNAs indeed play critical roles in PTC development and deserve our further investigations.

In this study, we first found that TR4 was increased in metastatic PTC patients compared to nonmetastatic controls. Overexpression of TR4 increased cell invasion/migration of PTC cells. Mechanistic dissection revealed that circ-FLNA/miR-149-5p/MMP9 signaling axis was involved in TR4-induced cell invasion/migration of PTC cells, which was also verified in xenografted mouse model. Together, our study builds strong rationale to develop TR4 targeted therapies towards metastatic PTC patients.

\section{Methods}

\section{Cell culture}

K-1, TPC-1 and 293T were purchased from Cell Bank in Chinese Academy of Sciences (Shanghai, China). $10 \%$ FBS DMEM (100 units $/ \mathrm{mL}$ penicillin, $100 \mu \mathrm{g} / \mathrm{mL}$ streptomycin) was used to culture cells in humidified $5 \% \mathrm{CO}_{2}$ environment at $37^{\circ} \mathrm{C}$.

\section{Lentivirus generation}


Lentivirus was generated as previously described. Briefly, $20 \mu \mathrm{g}$ plasmids with targeting sequence was cotransfected with psPAX2 $(10 \mu \mathrm{g})$ and pMD2.G $(\mu \mathrm{g})$ into 293T cells using the standard calcium phosphate transfection method. Lentivirus supernatant was collected with 0.45 filter after 48 hours and infected TC cells. $5 \mu \mathrm{g} / \mathrm{ml}$ puromycin was used to select shRNA infected TC cells.

\section{CircRNA construction}

Circ-FLNA nucleotides with $1 \mathrm{~kb}$ upstream and $200 \mathrm{bp}$ downstream were amplified from the gene locus, and cloned into PWPI (Pme I+ Pac I digested) using the Gibson ligation system at $50{ }^{\circ} \mathrm{C}$ for 1 hour. Gene mutation was performed using site-directed mutagenesis method as previously described.

\section{Real time qPCR}

Isolation of total RNAs was done with trizol reagent following the standard RNA extraction protocol. Two $\mu \mathrm{g}$ total RNAs were subjected to reverse transcription using superscript III transcriptase (Invitrogen). RT product with 200 dilutions was used to perform real time qRT-PCR in Bio-Rad CFX96 machine with SYBR green to determine the expression levels of interested genes. $18 \mathrm{~s}$ ribosome RNA was utilized to normalize gene expression.

RNase $\mathrm{R}$ digestion: RNase $\mathrm{R}$ digestion was done with $2 \mu \mathrm{g}$ total RNAs in $10 \mu$ reaction at $37^{\circ} \mathrm{C}$ for 1 hour: 0 unit or 20 units of RNase R (Epicentre), $1 \times$ RNase R buffer, 1 unit murine ribonuclease Inhibitor (NEB). Treated RNAs was used to perform reverse transcription to make cDNA. Then real time PCR was performed as aforementioned.

Western blotting: After PBS wash, TC cells were lysed by RIPA buffer and proteins were collected. $20 \mu \mathrm{g}$ proteins were loaded for electrophoresis in 10\%-12\% SDS-PAGE gel. Then proteins were transferred to PVDF membrane and blocked with 10\% BSA for 1 hour. Incubation with specific primary antibody was performed at $4{ }^{\circ} \mathrm{C}$ for at least 16 hours. Next day, the membrane was washed and incubated with 1:2500 HRP conjugated secondary antibody for 1 hour at room temperature. After extensive TBST wash, blots were visualized using enhanced chemiluminescence (Thermo Fisher Scientific).

\section{Wound healing assay}

TC cells with gene manipulations were scratched using a pipette tip. Images were captured at 0,24 hours. Experiment was done in triplicate and quantification was gained using the Image $\mathrm{J}$ software.

Transwell invasion assay: Transwell inserts (Corning) were pre-coated with 1:8 diluted matrigel (BD Bioscience) before use. PTC cells with gene manipulations were collected and loaded into the inserts at the density of $1 \times 10^{5} /$ well. $0 \%$ FBS medium in the lower chamber was used as chemoattractant to attract cells. 36 hours later, the invading cells in inserts were fixed with cold methanol and stained with $0.5 \%$ crystal violet. Images were taken by inverted microscope (Olympus) and quantifications of invading cells were done with ImageJ. 


\section{Chromatin Immunoprecipitation Assay (ChIP)}

TC cells were cross-linked with $4 \%$ formaldehyde for 10 minutes. Then cells were collected and sonicated to $300-500$ bp DNA fragments. Cell lysates were pre-cleared with protein A-agarose, and followed by the incubation with TR4 primary antibody (IgG was used as negative control). The next day, TR4-DNA fragments were intensively washed with high salt buffer. Proteins were digested with proteinase $\mathrm{K}$ and DNA fragments were extracted for RT-PCR. Specific primer sets were designed to amplify a target sequence within the promoter of FLNA and RT-PCR products were examined by agarose gel electrophoresis.

Xenografted mouse model: $2 \times 10^{6} \mathrm{~K}-1$ cells were subcutaneously implanted into 6-week male nude mice with 1:1 matrigel (Corning). Tumor size was measured weekly by caliper. 8 weeks later, mice were sacrificed and tumors were removed, washed, fixed for IHC staining.

Immunohistochemical staining (IHC): Tissues (human and mice) were fixed in $10 \%(\mathrm{v} / \mathrm{v})$ formaldehyde and embedded in paraffin. After hydration and antigen retrieval in boiling citrate buffer ( $\mathrm{pH} 6.0)$, sections were treated with $3 \%$ peroxidase in methanol for 15 minutes and blocked with $10 \%$ goat serum PBS buffer for 30 minutes. The slides were incubated with 1:100 diluted TR4 or MMP9 primary antibody at $4^{\circ} \mathrm{C}$ overnight. Next day, 1:100 diluted biotin-labeled secondary antibody was used to incubate slides for 30 minutes, and followed by 30 minutes incubation with streptavidin (PK-4000, Vectastain, Burlingame, CA.). The protein signal was determined by DAB staining.

\section{Statistical Analysis}

All statistical analyses were performed using GraphPad Prism software. Data were presented as mean \pm SE. Differences were analyzed with the one-way ANOVA test, and significance was set at $P<0.05$.

\section{Results}

\section{TR4 promotes cell invasion/migration of PTC cells}

The role of TR4 in PTC development has not yet been investigated. Therefore, we first examined TR4 expression in 20 pairs of primary PTC tumors and the paired lymph node metastatic PTC tumors. The data obtained from IHC staining demonstrated that TR4 expression level was significantly increased in metastatic PTC tumors compared to the paired primary PTC controls (Fig. 1A), which was consistent with the analysis from TCGA dataset showing that the level of TR4 was also remarkably increased in TC patients with nodal metastasis (Fig. 1B). More importantly, TC patients with high TR4 expression level tended to have shorter disease-free survival (DFS) time than patients with low TR4 expression level (Fig. 1C). These findings suggest that TR4 may play an oncogenic role in PTC development. To end this, we manipulated TR4 expression in K-1 and TPC-1 cells by knockdown or overexpression strategies and examined the invasive capacity of these cells. As shown in Fig. 1D-E, TR4 was successfully reduced by shRNAs in K-1 cells and overexpressed in TPC-1 cells. Knockdown of TR4 in K-1 cells led to a reduced cell 
invasion (Fig. 1F). On the contrary, cell invasion of TPC-1 was dramatically increased upon TR4 transfection (Fig. 1G). To double confirm it, we applied wound healing assay to monitor the cell migrating ability of PTC cells before and after TR4 manipulation. Data showed that TR4 depleted K-1 cells displayed a slower migrating rate than control K-1 cells (Fig. 1H). Consistently, TPC-1 cells with TR4 transfection had stronger capacity to migrate compared to the control TPC-1 cells (Fig. 11). Taken together, all these data suggest that TR4 drives PTC progression via promoting its cell migration/invasion.

\section{Circ-FLNA is one of factors contributing to TR4-mediated cell invasion/migration of PTC cells.}

To find the underlying mechanisms by which TR4 promotes cell invasion/migration of PTC cells, we turned our focus on circRNAs due to their potential roles in gene regulation. We first examined the expression levels of 10 circRNAs, which were differentially expressed between PTC tissues and the corresponding normal tissues according to the literature [19], in PTC cells before and after the manipulation of TR4. Results exhibited that four circRNAs (circRNA-0021553, circRNA-0079891, circRNA0091894 and circRNA-0004771) were consistently altered in K-1 and TPC-1 cells when we altered TR4 expression (Fig. 2A-B). To determine which circRNA was responsible for TR4 mediated cell invasion/migration of PTC cells, we applied rescue assay in TPC-1 cells. Interestingly, we found that TR4induced cell invasion of TPC-1 cells was reversed by circRNA-0091894 shRNAs but not by others (Fig. 2C). Wound healing assay also confirmed that knockdown of circRNA-0091894 blocked TR4induced cell migration of TPC-1 cells (Fig. 2D). As a back-splicing product of FLNA precursor (Fig. 2E), circRNA-0091894 was named as circ-FLNA for convenience and its circular property was confirmed by RNase R digestion using linear GAPDH mRNA as negative control (Fig. 2F). Next, we overexpressed circFLNA in K-1 cells (Fig. 2G) and found that induction of circ-FLNA could prevent shTR4 decreased cell invasion (Fig. 2H). Similarly, shTR4 reduced cell migration was diminished in K-1 cells in the presence of circ-FLNA transfection (Fig. 2l). Of note, we failed to observe any altered cell invasion of K-1 cells when FLNA was depleted (Supplementary Fig. 1), suggesting circ-FLNA but not its host gene contributed to cell invasion/migration of PTC cells. Taking together, we conclude that circ-FLNA is one of contributing factors responsible for TR4 mediated cell invasion/migration in PTC cells.

\section{Tr4 Transcriptionally Regulates Circ-flna}

As a transcription factor, TR4 could enter the nucleus and regulate numerous genes. We first looked at the promoter region of circ-FLNA host gene. As predicted in JASPAR, there were 6 TR4 binding elements (TR4E) at the $-2 \mathrm{~kb}$ promoter region of FLNA (Fig. 3A). To check which binding site was associated with the regulation of TR4 on circ-FLNA, we performed ChIP assay using TR4 antibody as a bait to pull down chromatin DNA fragments. The result revealed that TR4 could only bind TR4E-2/3 but not others (Fig. 3B), implying that TR4E-2/3 at the promoter region of FLNA may be tightly linked with the regulation of circ-FLNA by TR4. To confirm whether TR4 could directly affect the promoter's activity of FLNA and regulate circ-FLNA expression level, we cloned the wild type and mutated promoter region of FLNA, as 
depicted in Fig. 3C, into pGL3-basic backbone and examined their activities. As shown in Fig. 3D, overexpression of TR4 significantly increased the wild type promoter activity of FLNA in TPC-1 cells but had little effect on the mutate one. While, a significance decease of wild type promoter activity of FLNA was observed when TR4 was knocked down in K-1 cells, which was diminished when TR4-2/3 was mutated (Fig. 3E). Together, these results support the notion that TR4 transcriptionally regulates circFLNA expression level via directly binding to TR4E-2/3.

\section{Mechanistic dissection shows that miR-149-5p is involved in TR4/circ-FLNA mediated cell invasion/migration of PTC cells}

The sponge potential of circRNA in gene regulation has been wildly known. To find which miRNA was involved TR4/circ-FLNA mediated the progression of PTC cells, we pulled down circ-FLNA using biotin probe and detected its associated miRNAs. Result exhibited that miR-149-5p and miR-134-3p was evidently enriched with circ-FLNA (Fig. 4A). Rescue assay using miRNA inhibitor indicated that miR-149$5 p$ but not miR-134-3p participated into circ-FLNA mediated cell invasion of PTC cells, showing that a reduced cell invasion of TPC-1 cells by circ-FLNA knockdown was diminished in the presence of miR-149$5 p$ inhibitor (Fig. 4B). Similarly, inhibitory effect on cell migration of TPC-1 cells upon circ-FLNA knockdown was also lost when miR-149-5p inhibitor was supplemented into these cells (Fig. 4C). On the contrary, overexpression of miR-149-5p (Fig. 4D) could also block circ-FLNA induced cell invasion and cell migration of K-1 cells (Fig. 4E-F). Most importantly, TR4 induced cell invasion and cell migration of TPC-1 cells could also be attenuated when miR-149-5p was over-introduced into cells (Fig. 4G-H) while shTR4 failed to reduce cell invasion and cell migration of K-1 cells when cells were supplemented with miR-149$5 p$ inhibitor (Fig. 4I-J), implying the role of TR4 in PTC was also tightly connected with miR-149-5p. Furthermore, we also mutated the nucleotides of circ-FLNA responsible for miR-149-5p binding (Fig. 4K) and examined whether it affected the biological functions of circ-FLNA. Our results showed that the mutated form of circ-FLNA lost its ability to influence cell invasion and cell migration of K-1 cells (Fig. 4L$\mathrm{M})$, strengthening the point that the biological functions of circ-FLNA relied on its ability to interact with miR-149-5p. Together, all these data suggest that miR-149-5p is involved in TR4/circ-FLNA signal to regulate cell invasion/migration of PTC cells.

\section{MMP9 is the downstream effector controlling TR4/circ- FLNA/miR-149-5p induced cell invasion/migration of PTC cells}

To find the downstream targets contributing to TR4/circ-FLNA/miR-149-5p mediated cell invasion/migration of PTC cells, we examined the expression levels of several metastasis related genes (also predicated as miR-149-5p targeting genes) before and after the alteration of TR4 in TPC-1 and K-1 cells. Data showed that only MMP9 was consistently altered in TPC-1 and K-1 cells upon TR4 manipulation (Fig. 5A), implying MMP9 may be the candidate downstream effector responsible for TR4 induced cell invasion and migration of PTC cells. To test this postulation, we first examined MMP9 
expression level after the alteration of TR4/circ-FLNA/miR-149-5p signaling axis. Our data demonstrated that either circ-FLNA overexpression or miR-149-5p inhibition with inhibitor could reverse the reduction of MMP9 expression level by TR4 depletion in K-1 cells (Fig. 5B-C). In contrast, inhibition of circ-FLNA with shRNAs or miR-149-5p overexpression bore the capacity to prevent TR4 induced MMP9 expression level in TPC-1 cells (Fig. 5D-E). Most importantly, knockdown of MMP9 had capacity to impair TR4 induced cell invasion and cell migration of TPC-1 cells (Fig. 5F-G), suggesting MMP9 was involved in TR4/circFLNA/miR-149-5p signal to control cell invasion/migration of PTC cells. In fact, analyses from TCGA dataset indicated that MMP9 expression level was correlated with TC development. MMP9 expression level was significantly increased in TC tissues compared to normal tissues (Fig. 5H). DFS analysis also showed that MMP9 was a poor prognostic factor in TC progression ( $p=0.017$, Figure I). And TC patients with T3 + T4 stage or metastasis tended to express higher expression level of MMP9 compared to the corresponding controls (Supplementary Fig. 2A-C). Interestingly, this trend only existed in TC patients with age above 55 (Supplementary Fig. 2A-C). To further confirm MMP9 was a direct target of miR-149-5p, we constructed wild type and mutated 3'-UTR of MMP9 into the psi-CHECK 2 enhancer and tested the luciferase activity upon the manipulation of miR-149-5p (Fig. 5J). Our results demonstrated that miR-149$5 p$ could significantly suppress the luciferase activity of wild type but not the mutated 3'-UTR of MMP9 in TPC-1 cells (Fig. 5K). Consistent result was gained from K-1 cells, revealing that miR-149-5p inhibitor remarkably enhanced the wild type luciferase activity of 3'-UTR of MMP9 (Fig. 5L). However, miR-149-5p inhibitor failed to affect the luciferase activity of mutate 3'-UTR of MMP9 (Fig. 5L). Taking together, we conclude that MMP9 is a direct target of miR-149-5p and accounts for TR4/circ-FLNA/miR-149-5p mediated cell invasion and migration of PTC cells.

\section{Xenografted mouse model confirms the role of TR4/circ- FLNA signal in PTC progression}

Next, we applied xenografted mouse model to verify the role of TR4/circ-FLNA in PTC progression. We subcutaneously implanted $1 \times 10^{6} \mathrm{~K}-1$ cells with the following gene manipulations: 1) pWPI + pLKO; 2) TR4 + pLKO; 3) pWPI + shcirc-FLNA; 4) TR4 + shcirc-FLNA, under the skin of 6-week male nude mice and monitored tumor growth. Data showed that TR4 evidently promoted K-1 tumor growth while knockdown of circ-FLNA could suppress K-1 tumor growth (Fig. 6A). And TR4-induced K-1 tumor growth was attenuated when circ-FLNA was depleted by shRNAs (Fig. 6A), strengthening the role of TR4/circ-FLNA signal in papillary thyroid tumor growth. The curves of tumor volume also confirmed the data (Fig. 6B). As the downstream effector of TR4/circ-FLNA/miR-149-5p, MMP9 expression level was examined by IHC staining in various K-1 tumors, showing that TR4 significantly promoted the MMP9 expression level and circ-FLNA knockdown could prevent TR4 induced MMP9 expression (Fig. 6C). Together, these in vivo results support the oncogenic role of TR4/circ-FLNA/miR-149-5p/MMP9 signal in papillary thyroid tumor development.

To summarize, TR4 played a tumor promoting role in PTC development by controlling circ-FLNA/miR-149$5 \mathrm{p} / \mathrm{MMP9}$ signal to regulate cell invasion and migration. 


\section{Discussion}

A small portion of PTC will progress to advanced stage with distant metastasis, which has little response to either radioactive iodide treatment or hormone suppressive therapy [1]. Therefore, understanding the molecular biology of metastatic PTC has chance to develop novel targeted therapies towards advanced PTC patients. In this study, we first found that TR4 was significantly increased in PTC patients with distant metastasis compared to patients without metastasis, which was consistent with the analyses from TCGA dataset. Mechanistic study demonstrated that TR4 could transcriptionally regulate circ-FLNA expression level via directly binding to its upstream promoter, which served as a sponge of miR-149-5p to enhance MMP9 expression. Our identified TR4/circ-FLNA/miR-149-5p/MMP9 axis can promote cell invasion/migration of PTC cells. Furthermore, the oncogenic roles of TR4 and circ-FLNA were also verified in xenografted mouse model. Overall, our study defines the role of TR4 in PTC development and targeting TR4 may be an alternative option for metastatic PTC patients.

The tumor promoting role of TR4 has been documented in various cancers [9-13], promoting scientists to identify TR4 inhibitor for better treatment of cancers. Therefore, it is of importance and interest to develop small molecules which can specifically inhibit TR4 activity. Previous study showed that metformin, a clinical drug used in patient with diabetes type II, can suppress TR4 transactivation via triggering AMPK signaling [20]. Given the fact that TR4 is overexpressed in metastatic PTC patients, it is reasonable to test the effect of metformin on PTC cell invasion and to prompts the clinical trial of metformin in metastatic PTC patients. In addition, a small molecule, tretinoin, was also reported to suppress RCC progression and affect sunitinib sensitivity via directly inhibiting the function of TR4 [21]. As fact, the potential anti-cancer role of tretinoin in thyroid cancer has been recognized many decades ago. It is possible that the anti-cancer effect of tretinoin on thyroid cancer relies on its inhibition on TR4's function.

The pathological and physiological roles of noncoding RNA including circRNAs have been increasing noticed recently [22-26]. Our data showed that circ-FLNA acted as a sponge of miR-149-5p to increase MMP9 expression, leading to enhanced cell invasion of PTC cells. However, how to target circRNA remains a scientific concern because one circRNA may interact with several miRNAs and one miRNA has hundreds of targets. Although we defined the role of circ-FLNA in TC cells, the translational value of circFLNA is currently limited.

Numerous studies have demonstrated that MMP proteins are tightly associated with cancer initiation and progression [27-29]. As an important member of MMP family, MMP9 was shown as the downstream effector of TR4/circ-FLNA/miR-149-5p to control PTC cell invasion/migration. Importantly, MMP9 was highly expressed in metastatic PTC patients and served as a poorly prognostic factor for DFS according to TCGA dataset, suggesting targeting MMP9 may be a promising therapeutic strategy towards metastatic PTC patients. In fact, clinical trials of MMP inhibitors have been examined in various cancers many decades ago. However, the results were disappointing due to the severe side effects of MMP inhibitors [30]. Recently, new generation of MMP inhibitors has been developed after the better 
understanding of MMP structure [31]. Preclinical trials suggested that these new MMP inhibitors have improved toxicity and better efficacy. Therefore, it will be promising to see the therapeutic efficacy of new generation of MMP9 inhibitor towards metastatic PTC patients.

\section{Conclusions}

In summary, our results proved the oncogenic role of TR4 in PTC development. TR4 promoted PTC cell invasion/migration via increasing circ-FLNA level, which worked as a miR-149-5p sponge to increase MMP9 expression level. TR4/circ-FLNA/miR-149-5p/MMP9 signal may be an ideal therapeutic target for metastatic PTC patients.

\section{Declarations}

\section{Ethics approval and consent to participate}

The study was carried out under the approval of the ethics committee of Xiangya Hospital Central South University and followed the Interdisciplinary Principles and Guidelines for the Use of Animals in Research, Testing, and Education by the New York Academy of Sciences, Ad Hoc Animal Research Committee.

\section{Consent for publication}

Not applicable.

\section{Availability of data and materials}

Not applicable.

\section{Competing interests}

The authors declare that they have no competing interests.

\section{Funding}

This work was supported by National Natural Science Foundation of China (81903004).

\section{Acknowledgements}

We thanked Thelma for editing the manuscript for us.

\section{References}

1. Jin S, Borkhuu O, Bao W, Yang YT. Signaling Pathways in Thyroid Cancer and Their Therapeutic Implications. J Clin Med Res. 2016;8(4):284-296.

2. Siegel RL, Miller KD, Jemal A. Cancer statistics, 2019. CA Cancer J Clin. 2019;69(1):7-34. 
3. La Vecchia C, Malvezzi M, Bosetti C, Garavello W, Bertuccio P, Levi F, et al. Thyroid cancer mortality and incidence: a global overview. Int J Cancer. 2015;136(9):2187-2195.

4. Rivera M, Ghossein RA, Schoder H, Gomez D, Larson SM, Tuttle RM. Histopathologic characterization of radioactive iodine-refractory fluorodeoxyglucose-positron emission tomography-positive thyroid carcinoma. Cancer. 2008;113(1):48-56.

5. Sáez JM. Treatment directed to signalling molecules in patients with advanced differentiated thyroid cancer. Anticancer Agents Med Chem. 2013;13(3):483-495.

6. Chang C, Da Silva SL, Ideta R, Lee Y, Yeh S, Burbach JP. Human and rat TR4 orphan receptors specify a subclass of the steroid receptor superfamily. Proc Natl Acad Sci U S A. 1994;91(13):6040-6044.

7. Collins LL, Lee YF, Ting HJ, Lin WJ, Liu NC, Meshul CK, et al. The roles of testicular nuclear receptor 4 (TR4) in male fertility-priapism and sexual behavior defects in TR4 knockout mice. Reprod Biol Endocrinol. 2011;9:138.

8. Lin SJ, Ho HC, Lee YF, Liu NC, Liu S, Li G, et al. Reduced osteoblast activity in the mice lacking TR4 nuclear receptor leads to osteoporosis. Reprod Biol Endocrinol. 2012;10:43.

9. Bai J, Yeh S, Qiu X, Hu L, Zeng J, Cai Y, et al. TR4 nuclear receptor promotes clear cell renal cell carcinoma (ccRCC) vasculogenic mimicry (VM) formation and metastasis via altering the miR4903p/vimentin signals. Oncogene. 2018;37(44):5901-5912.

10. Wang M, Sun Y, Xu J, Lu J, Wang K, Yang DR, et al. Preclinical studies using miR-32-5p to suppress clear cell renal cell carcinoma metastasis via altering the miR-32-5p/TR4/HGF/Met signaling. Int $J$ Cancer. 2018;143(1):100-112.

11. Lin SJ, Yang DR, Li G, Chang C. TR4 Nuclear Receptor Different Roles in Prostate Cancer Progression. Front Endocrinol (Lausanne). 2015;6:78.

12. Lin SJ, Lee SO, Lee YF, Miyamoto H, Yang DR, Li G, et al. TR4 nuclear receptor functions as a tumor suppressor for prostate tumorigenesis via modulation of DNA damage/repair system. Carcinogenesis. 2014;35(6):1399-1406.

13. Jin R, Lin H, Li G, Xu J, Shi L, Chang C, et al. TR4 nuclear receptor suppresses HCC cell invasion via downregulating the EphA2 expression. Cell Death Dis. 2018;9(3):283.

14. Ashwal-Fluss R, Meyer $M$, Pamudurti NR, Ivanov A, Bartok $O$, Hanan $M$, et al. circRNA biogenesis competes with pre-mRNA splicing. Mol Cell. 2014;56(1):55-66.

15. Kristensen LS, Hansen TB, Ven $\varnothing$ MT, Kjems J. Circular RNAs in cancer: opportunities and challenges in the field. Oncogene. 2018;37(5):555-565.

16. Holdt LM, Kohlmaier A, Teupser D. Molecular functions and specific roles of circRNAs in the cardiovascular system. Noncoding RNA Res. 2018;3(2):75-98.

17. Ye M, Hou H, Shen M, Dong S, Zhang T. Circular RNA circFOXM1 Plays a Role in Papillary Thyroid Carcinoma by Sponging miR-1179 and Regulating HMGB1 Expression. Mol Ther Nucleic Acids. 2020;19:741-750. 
18. Cai X, Zhao Z, Dong J, Lv Q, Yun B, Liu J, et al. Circular RNA circBACH2 plays a role in papillary thyroid carcinoma by sponging miR-139-5p and regulating LMO4 expression. Cell Death Dis. 2019;10(3):184.

19. Peng N, Shi L, Zhang Q, Hu Y, Wang N, Ye H. Microarray profiling of circular RNAs in human papillary thyroid carcinoma. PLoS One. 2017;12(3):e0170287.

20. Kim E, Liu NC, Yu IC, Lin HY, Lee YF, Sparks JD, et al. Metformin inhibits nuclear receptor TR4mediated hepatic stearoyl-CoA desaturase 1 gene expression with altered insulin sensitivity. Diabetes. 2011;60(5):1493-1503.

21. Shi H, Sun $Y$, He M, Yang X, Hamada M, Fukunaga T, et al. Targeting the TR4 nuclear receptormediated IncTASR/AXL signaling with tretinoin increases the sunitinib sensitivity to better suppress the RCC progression. Oncogene. 2020;39(3):530-545.

22. Zhong Y, Du Y, Yang X, Mo Y, Fan C, Xiong F, et al. Circular RNAs function as ceRNAs to regulate and control human cancer progression. Mol Cancer. 2018;17(1):79.

23. Wang Y, et al. Circular RNAs in human cancer. Mol Cancer. 2017;16(1):25.

24. Wang Y, Mo Y, Gong Z, Yang X, Yang M, Zhang S, et al. Circular RNA DOCK1 promotes bladder carcinoma progression via modulating circD0CK1/hsa-miR-132-3p/Sox5 signalling pathway. Cell Prolif. 2019;52(4):e12614.

25. Dong Y, He D, Peng Z, Peng W, Shi W, Wang J, et al. Circular RNAs in cancer: an emerging key player. J Hematol Oncol. 2017;10(1):2.

26. Bai N, Peng E, Qiu X, Lyu N, Zhang Z, Tao Y, et al. circFBLIM1 act as a ceRNA to promote hepatocellular cancer progression by sponging miR-346. J Exp Clin Cancer Res. 2018;37(1):172.

27. Kessenbrock K, Plaks V, Werb Z. Matrix metalloproteinases: regulators of the tumor microenvironment. Cell. 2010;141(1):52-67.

28. Liotta LA, Tryggvason K, Garbisa S, Hart I, Foltz CM, Shafie S. Metastatic potential correlates with enzymatic degradation of basement membrane collagen. Nature. 1980;284(5751):67-68.

29. Winer A, Adams S, Mignatti P. Matrix Metalloproteinase Inhibitors in Cancer Therapy: Turning Past Failures Into Future Successes. Mol Cancer Ther. 2018;17(6):1147-1155.

30. Coussens LM, Fingleton B, Matrisian LM. Matrix metalloproteinase inhibitors and cancer: trials and tribulations. Science. 2002;295(5564):2387-2392.

31. Fields GB. The Rebirth of Matrix Metalloproteinase Inhibitors: Moving Beyond the Dogma. Cells. 2019;8(9):984.

\section{Figures}




\section{Figure 1}

A
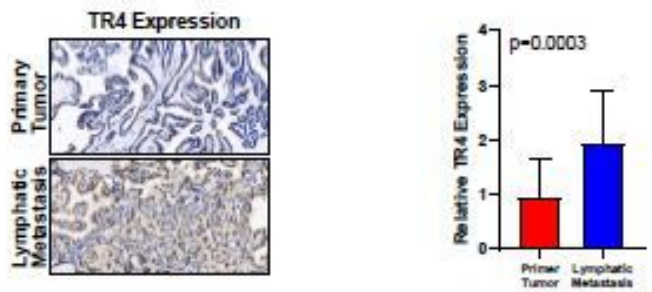

B

C
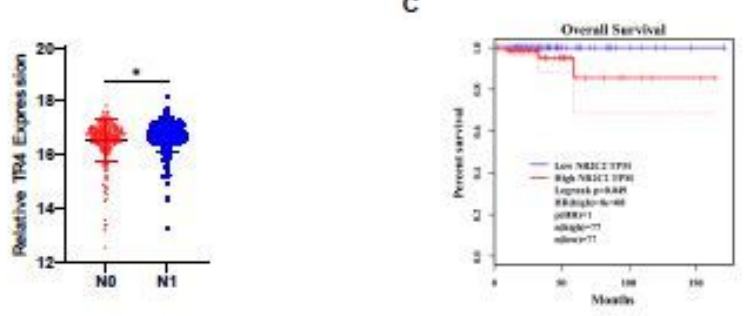

D

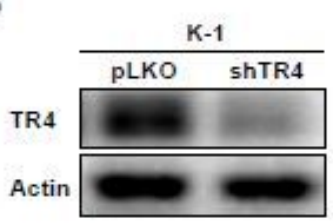

E

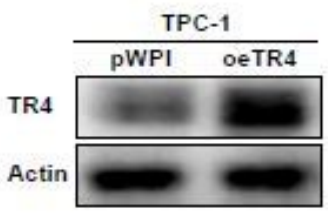

F

G
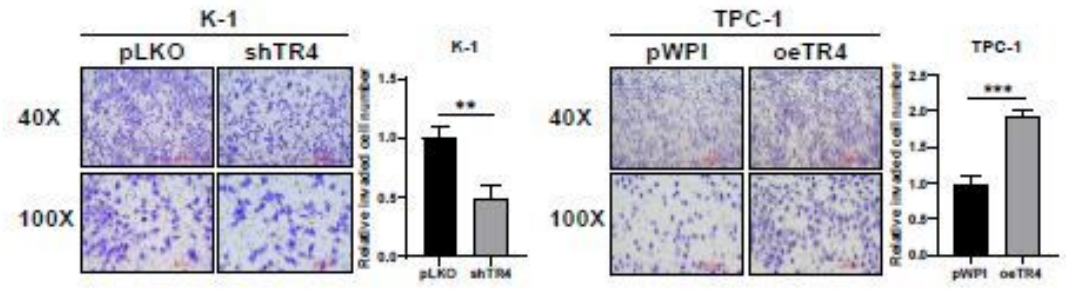

H
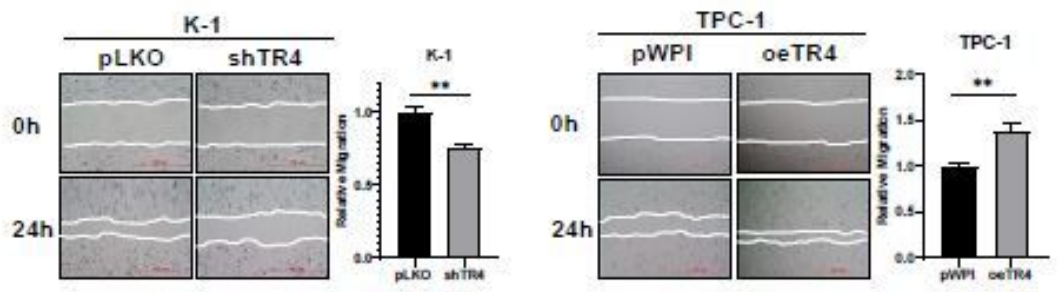

\section{Figure 1}

TR4 promotes cell invasion/migration of PTC cells. A. TR4 expression level was examined in 20 pairs of primary PTC tumors and the paired lymph node metastatic PTC tumors using IHC staining strategy. Left, representative images of TR4 signal in PTC patients: Top, image from primary PTC tumor; bottom, image from the paired PTC tumors metastasizing into lymph node. Right, quantification of TR4 signal in PTC patients. Paired T test was used to make the statistical analysis. B. TR4 expression level was significantly 
increased in N1 TC patients compared to N0 TC patients. N1, nodal metastasis. N0, non-metastasis. C. DFS analysis of TC patients classified by TR4 expression level. D. The efficiency of TR4 knockdown in K1 cells. Actin was internal control. E. The efficiency of TR4 overexpression in TPC-1 cells. Actin was loading control. F. Knockdown of TR4 suppressed cell invasion of K-1 cells. Left, representative images of invading K-1 cells. Right, statistical analysis of invading K-1 cells. G. Overexpression of TR4 promoted cell invasion of TPC-1 cells. Left, representative images of invading TPC-1 cells. Right, statistical analysis of invading TPC-1 cells. H. Wound healing assay showed that knockdown of TR4 inhibited cell migration of K-1 cells. Left, representative images of migrating K-1 cells. Right, statistical analysis of migrating K-1 cells. I. Wound healing assay revealed that TR4 overexpression enhanced the migrating ability of TPC-1 cells. Left, representative images of migrating TPC- 1 cells. Right, statistical analysis of migrating TPC-1 cells. ${ }^{*} P<0.05,{ }^{\star \star} P<0.01,{ }^{\star \star \star} P<0.001, N S=$ no significance. 


\section{Figure 2}

A

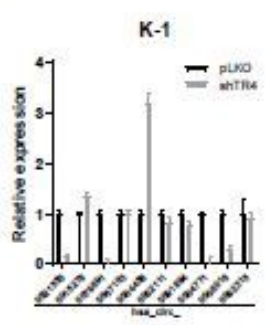

B

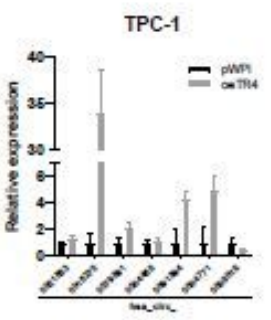

c

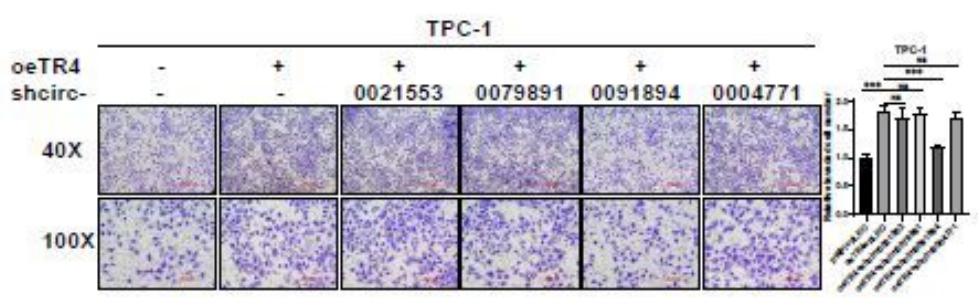

D

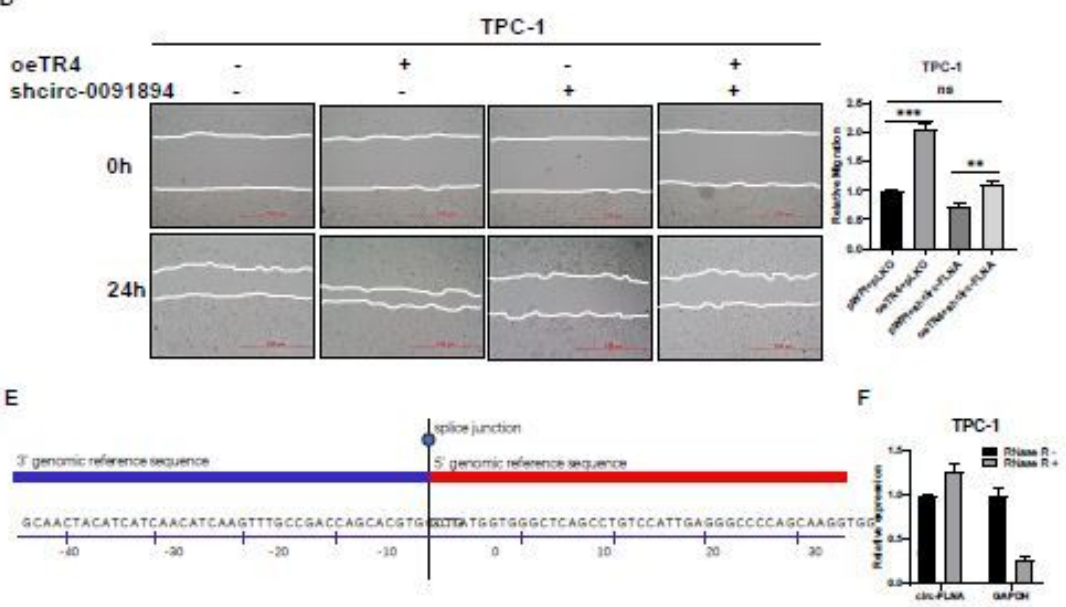

G

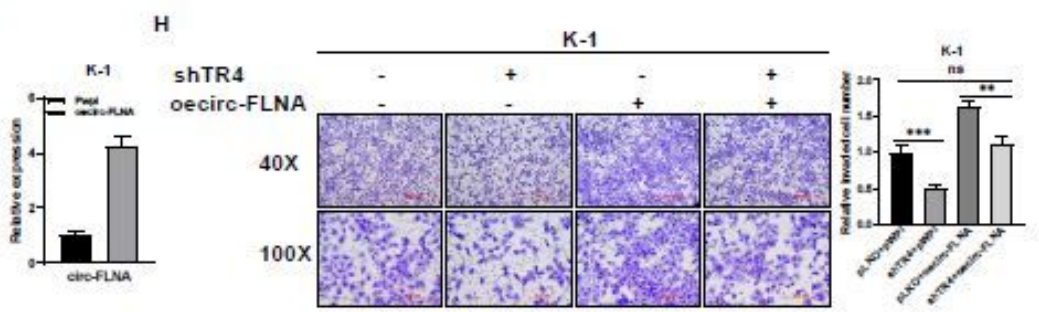

1

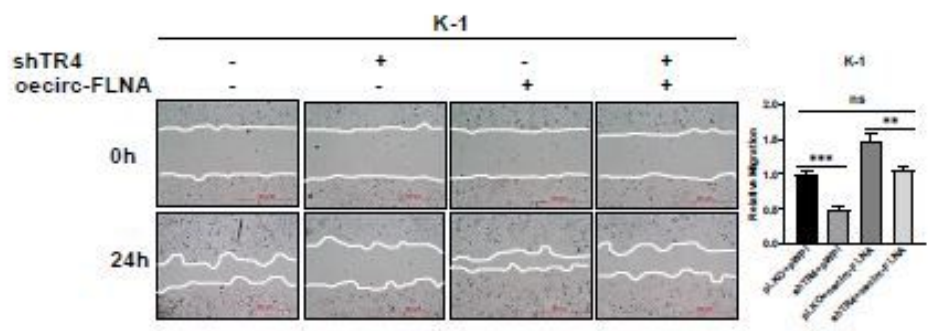

\section{Figure 2}

Circ-FLNA is one of factors contributing to TR4-mediated cell invasion/migration of PTC cells. A. The expression levels of circRNAs in K-1 cells before and after TR4 knockdown. GAPDH was used to normalize gene expression. B. The expression levels of circRNAs in TPC-1 cells before and after TR4 overexpression. GAPDH was used to normalize gene expression. C. Transwell invasion assay showed that circRNA0091894 knockdown could prevent TR4-induced cell invasion of TPC-1 cells. Left, 
representative images of invading TPC-1 cells. Right, statistical analysis of invading TPC-1 cells. D. Wound healing assay revealed that TR4-induced cell migration of TPC-1 cells was reversed by shcirc0091894. Left, representative images of migrating TPC-1 cells. Right, statistical analysis of migrating TPC-1 cells. E. Nucleotide Sequence of circ-FLNA. F. The circular property of circ-FLNA was confirmed by RNase R digestion. GAPDH served as negative control. G. The efficiency of overexpressing circ-FLNA in K1 cells. H. Overexpression of circ-FLNA could block shTR4 reduced cell invasion of K-1 cells. Left, representative images of invading K-1 cells. Right, statistical analysis of invading K-1 cells. I. Overexpression of circ-FLNA could also prevent shTR4 reduced cell migration of K-1 cells. Left, representative images of migrating $\mathrm{K}-1$ cells. Right, statistical analysis of migrating $\mathrm{K}-1$ cells. ${ }^{\star \star} \mathrm{P}<0.01$, *** $\mathrm{P}<0.001, \mathrm{NS}=$ no significance.

\section{Figure 3}

A

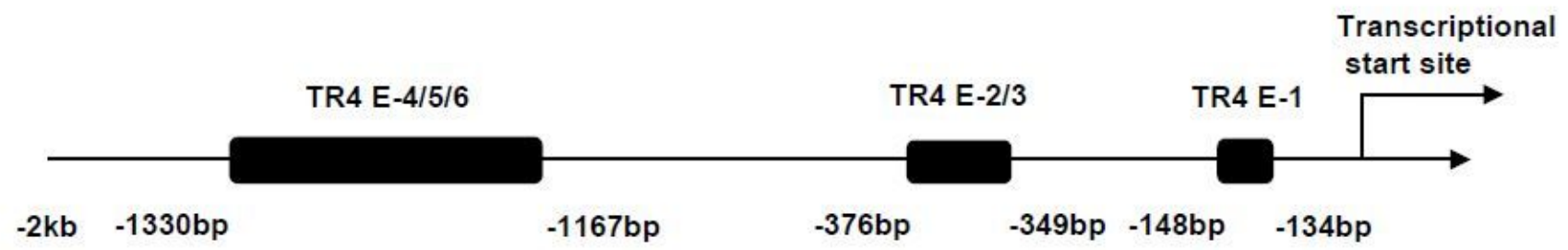

B

C

TR4 E-1

TR4 E-2/3

TR4 E-4/5/6

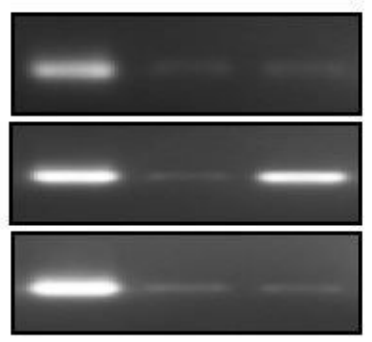

D

PTC-1

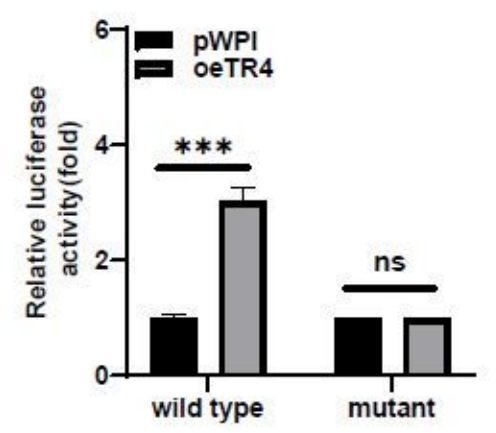

K-1

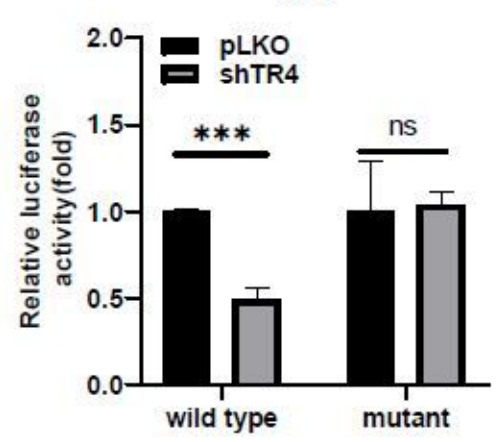

Figure 3 
TR4 transcriptionally regulates circ-FLNA level. A. Six TR4 binding sites were predicted in the upstream promoter of circ-FLNA. B. ChIP assay exhibited that TR4 bond to TR4E-2/3 in the upstream promoter of circ-FLNA. C. Schematic depiction showed that how wild type and mutated circ-FLNA's promoter were constructed. D. Luciferase activities of wild type and mutated circ-FLNA's promoter before and after TR4 overexpression in PTC-1 cells. E. Luciferase activities of wild type and mutated circ-FLNA's promoter before and after TR4 depletion in K-1 cells. ${ }^{* *} \mathrm{P}<0.001$, NS=no significance.

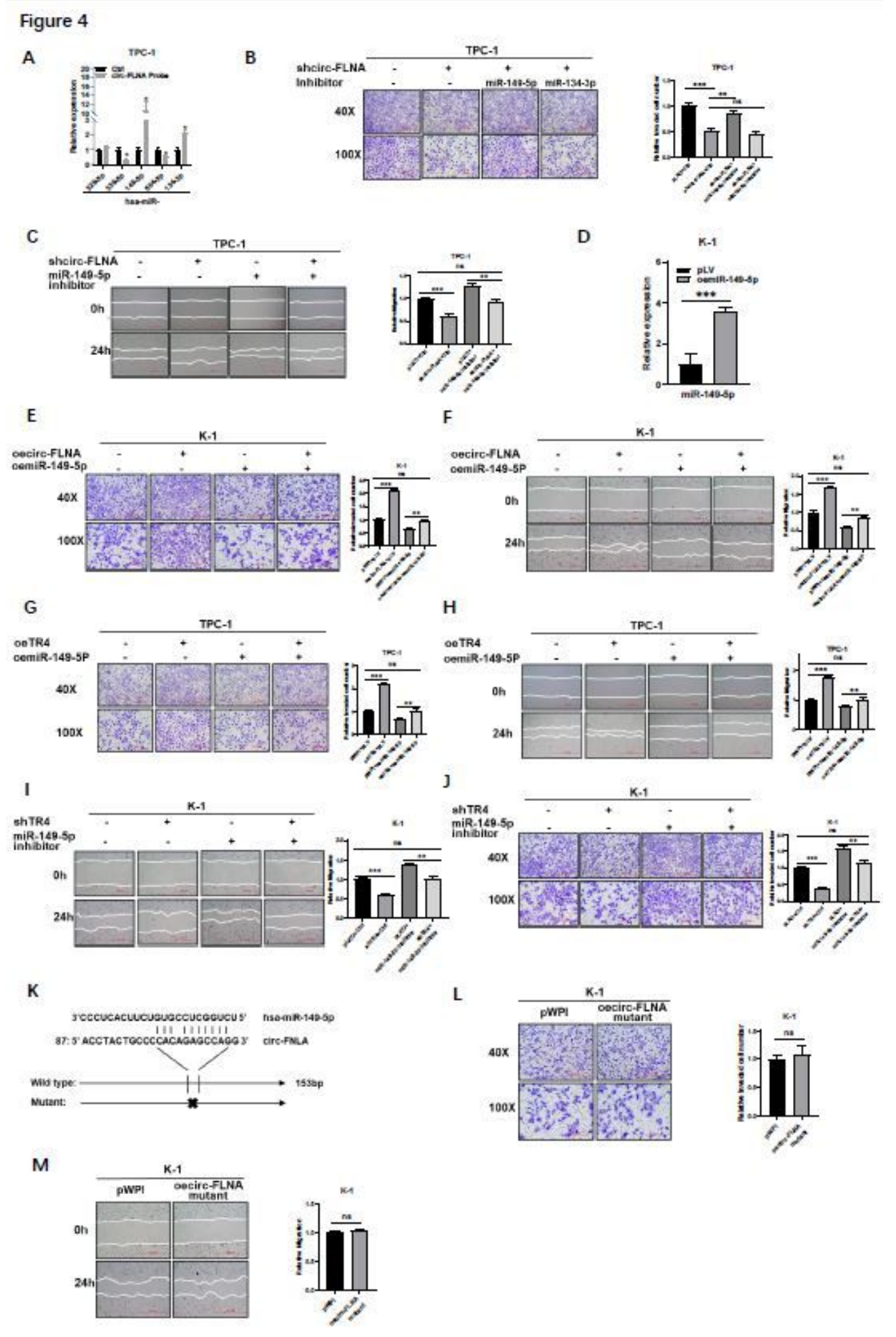

Figure 4 
Mechanistic dissection shows that miR-149-5p is involved in TR4/circ-FLNA mediated cell invasion/migration of PTC cells. A. miR-149-5p and miR-134-3p were associated with circ-FLNA. B. Inhibition of miR-149-5p reversed shcirc-FLNA reduced cell invasion of TPC-1 cells. Left, representative images. Right, quantification of invading cells. C. Inhibition of miR-149-5p reversed shcirc-FLNA reduced cell migration of TPC-1 cells. Left, representative images. Right, quantification of migrating cells. D. The efficiency of miR-149-5p transfection in K-1 cells. E-F. Overexpression of miR-149-5p inhibited cell invasion (E) and cell migration (F) of K-1 cells induced by circ-FLNA. Left, representative images. Right, quantification of invading or migrating cells. G-H. Overexpression of miR-149-5p inhibited cell invasion (G) and cell migration $(\mathrm{H})$ of $\mathrm{K}-1$ cells induced by TR4. Left, representative images. Right, quantification of invading or migrating cells. I-J. Knockdown of TR4 failed to affect cell migration (I) and cell invasion (J) of K-1 cells in the presence of miR-149-5p inhibitor. Left, representative images. Right, quantification of invading or migrating cells. K. The predicted binding nucleotides between miR-149-5p and circ-FLNA. L-M. Mutated form of circ-FLNA failed to affect cell invasion ( $L$ ) and cell migration (M) of K-1 cells. Left, representative images. Right, quantification of invading or migrating cells. ${ }^{*} P<0.01,{ }^{*} * *<0.001, N S=$ no significance. 


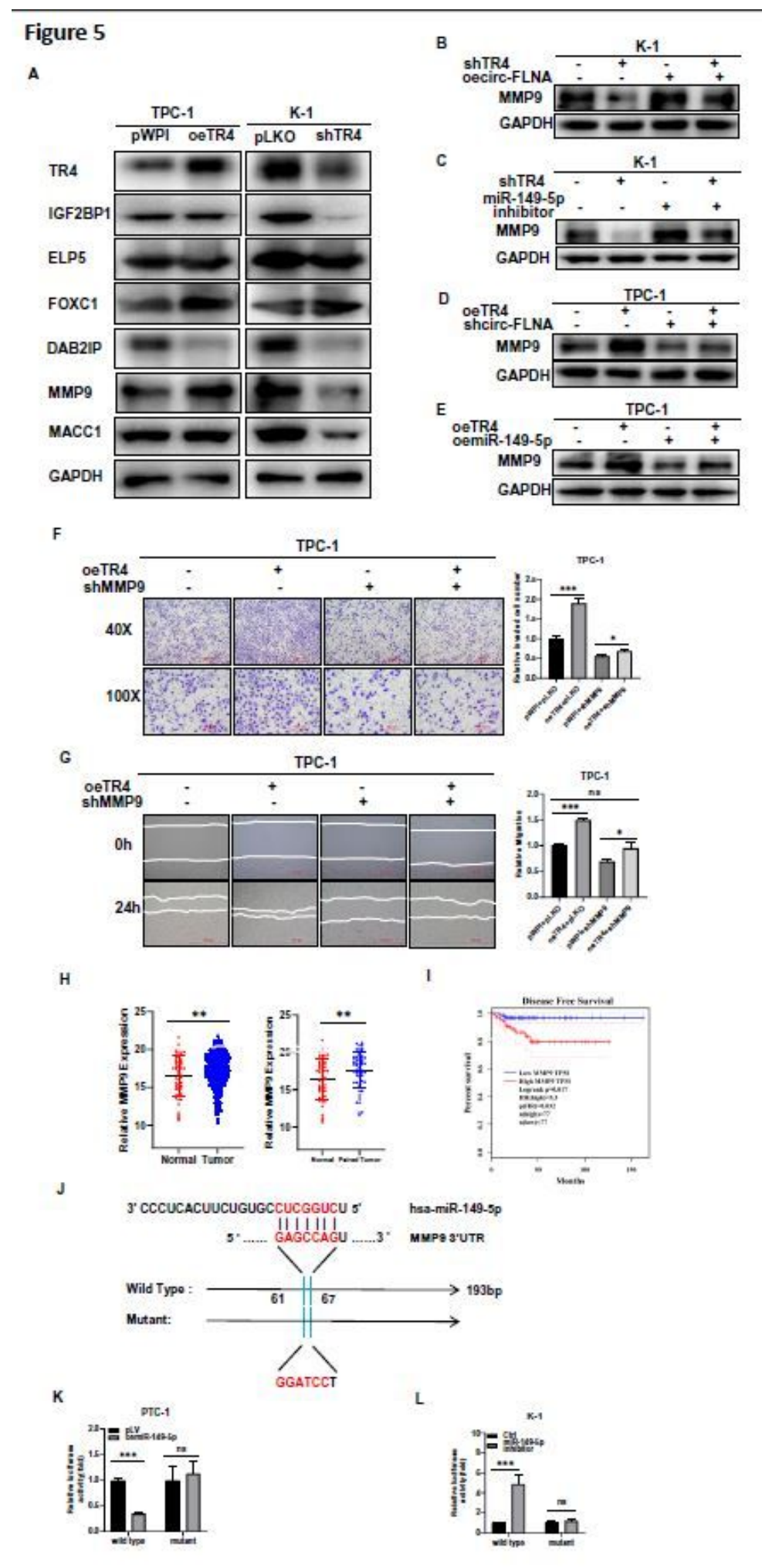

\section{Figure 5}

MMP9 is the downstream effector controlling TR4/circ-FLNA/miR-149-5p induced cell invasion/migration of PTC cells. A. Several genes as predicated as miR-149-5p were detected by western blotting before and after TR4 manipulation in K-1 cells and TPC-1 cells. GAPDH was served as internal control. B-C. Overexpression of circ-FLNA (B) or inhibition of miR-149-5p (C) could block shTR4 reduced MMP9 expression by western blotting analysis. GAPDH was loading control. D-E. Knockdown of circ-FLNA (E) or 
miR-149-5p (D) attenuated TR4 induced MMP9 expression by western blotting analysis. GAPDH was internal control. F-G. TR4 induced cell invasion (F) and cell migration (G) of TPC-1 cells were attenuated when MMP9 was knocked down. Left, representative images. Right, quantification of invading or migrating cells. H. MMP9 was significantly increased in TC tissues compared to the corresponding controls. Left, normal vs tumor. Right, TC tissues vs paired adjacent normal tissues. I. DFS analysis of TC patients classified by MMP9 expression level. J. Schematic Depiction of how wild type and mutated 3'UTR of MMP9 were constructed. K. The luciferase activity of wild type but not mutated 3'UTR of MMP9 was reduced by miR-149-5p in TPC-1 cells. L. The luciferase activity of wild type but not mutated 3'UTR of MMP9 was enhanced by miR-149-5p inhibitor in $\mathrm{K}-1$ cells. ${ }^{*} \mathrm{P}<0.01, * * * \mathrm{P}<0.001$, NS=no significance.

\section{Figure 6}

A

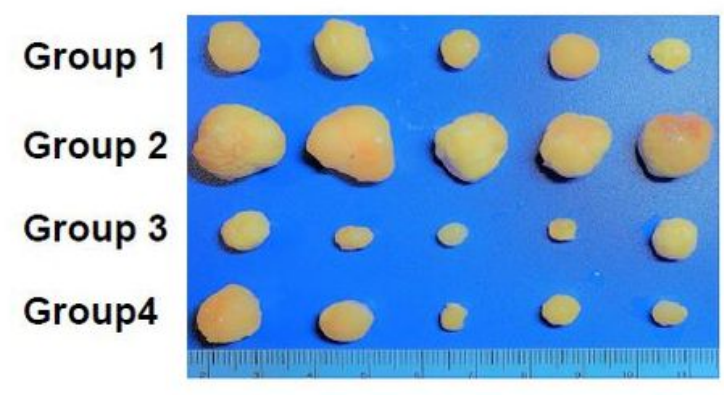

B

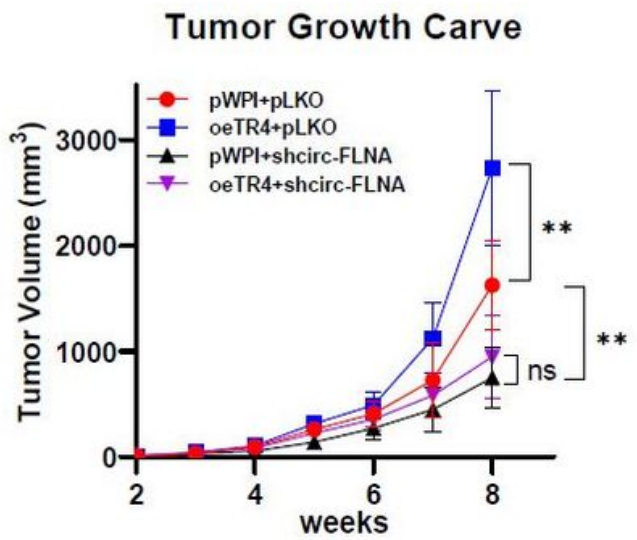

C
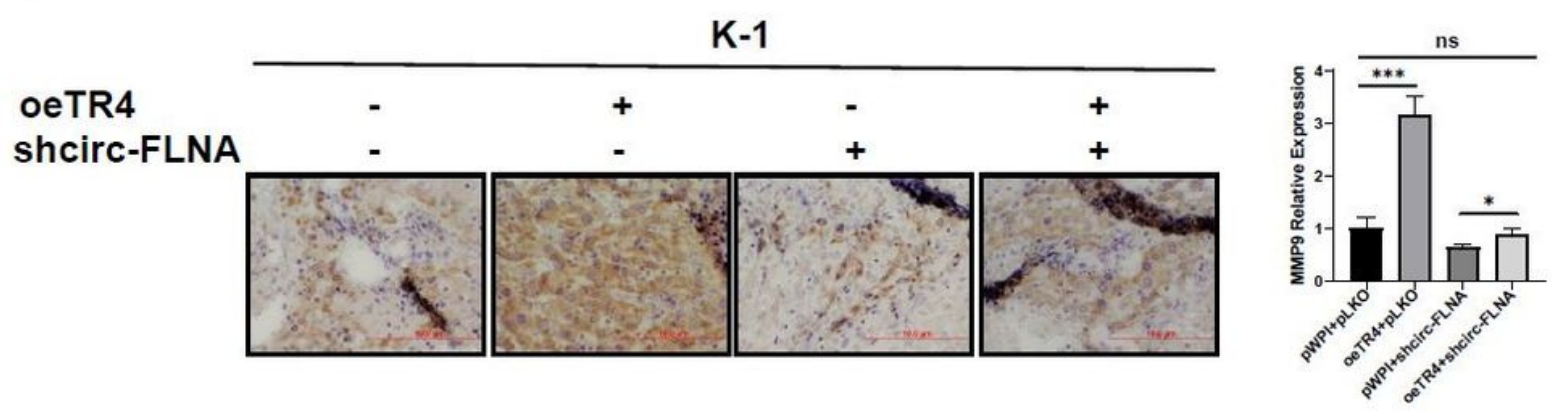

Figure 6

Xenografted mouse model confirms the role of TR4/circ-FLNA signal in PTC progression. A. K-1 tumor growth was weekly monitor. B. The growth curve of tumor volume was showed. Tumor volume=length* (width*width)/2. C. Representative image of MMP9 signal in K-1 tumors. ${ }^{\star} P<0.05,{ }^{* \star} P<0.01,{ }^{\star * *} P<0.001$, NS=no significance. 